\title{
Energy estimation of the interaction of a roller vibratory classifier's blade with crushed rock mass for solving design problems
}

\author{
Volodymyr Nadutyi ${ }^{1}$, Valentyna Chelyshkina ${ }^{1, *}$, Vitalii Sukhariev ${ }^{1}$, and Jamil Haddad $^{2}$ \\ ${ }^{1}$ Institute of Geotechnical Mechanics named by N. Poljakov of National Academy of Sciences of \\ Ukraine, 49095, Dnipro, Simferopolska Str., 2a, Ukraine \\ ${ }^{2}$ Al-Balqa Applied University, 11134, Amman, Marka Ave., Jordan
}

\begin{abstract}
Annotation. The roller vibratory classifier is equipped with an impact device for implementing vibro-shock mode, which improves the classification efficiency, especially of viscous and wet materials. The new device is a disk nozzle with blades, which are made like petals of wearresistant polymer. The immersion of a petal at a given depth in a crushed rock mass is investigated experimentally. Theoretical studies includes pair correlation and regression analysis methods. It is obtained that an increase in humidity promotes penetration and leads to a decrease in the energy necessary for impact, whereas an increase in particle size and layer thickness leads to its increase. Analysis of regression equations makes it possible to establish the percentage ratio of influencing factors. The article presents mathematical models of impact energy from variable factors for the initial moisture content of the material, including separately for small classes $(-2.5+1) \mathrm{mm}$ and further, with variation of all three parameters in the range: layer thickness $-20-40 \mathrm{~mm}$, particle size $1-10 \mathrm{~mm}$, humidity up to $9 \%$. The obtained correlation dependencies and regression models are necessary for improving the existing and designing new blade-type roller vibratory classifiers.
\end{abstract}

\section{Introduction}

The improvement of roller vibratory classifier is promising using additional shock loads, which makes it possible to implement vibration-shock mode and active mixing of the material. Due to this, the adhesion forces between the particles are violated, which leads to an increase in the classification efficiency, especially of viscous and wet materials [1]. To create shock loads, it is proposed to made blade-type roller vibratory classifiers. It is proposed to place a rim (disk) on the cylindrical rolls of the classifier with the blades, which are made like petals. The blades fixed on it in the form of teeth. Discs with blades (Figure 1) are made of hydrophobic polymers, for example, wear-resistant (tread) rubbers $[1,2]$.

Roller vibratory classifier with the following parameters were experimentally tested: roll diameter $-80-100 \mathrm{~mm}$, rim thickness for attaching the blades $25-30 \mathrm{~mm}$, one blade

${ }^{*}$ Corresponding author: chel.valenti@gmail.com 
height 45-50 mm, roll speed 15-17 r/s [3, 4].

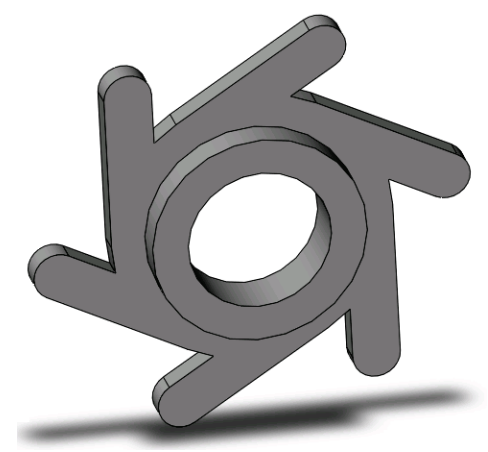

Fig. 1. Impact device configuration with polymer blades for the roller vibratory classifiers.

At the edge of the tooth, without taking into account the displacement of the center of mass due to vibration, the linear velocity is $2 \cdot 3.14 \cdot(0.05+0.025+0.045) \cdot 5=11.3(\mathrm{~m} / \mathrm{s})$. With a blade mass of $0.2-0.3 \mathrm{~kg}$, the kinetic energy of rotation in the air is approximately 13-19 J.

For example, household jackhammers have an impact energy of $5 \mathrm{~J}$. That is, in a rough estimate, there is enough energy at the end of the blade (petal) to overcome the resistance of the medium and immerse it in the rock mass. Due to this, large pieces are cleaned of adhering small fractions, which, in turn, are actively mixed. This allows for a more efficient classification of raw materials, especially wet and sticky materials.

However, the mechanical energy during the movement of the blade in the rock mass is significantly reduced due to scattering during vibration and the cost of overcoming the forces of external and internal friction. Note that for blades made of rubber-based polymer, internal friction plays a significant role. Here, under the action of an external force, highly elastic deformation occurs - parts of the chain (segments) of the macromolecules are displaced, which, after the termination of the action of an external force, again return to the folded state due to thermal motion. However, the manufacture of blades from wear-resistant rubbers is preferable in view of a longer service life compared to, for example, metal ones.

Taking into account the indicated phenomena of dissipation, the theoretical calculation of the energy required for the penetration of the blade into the rock layer is a difficult task. Therefore, an approximate model was considered, namely, the energy necessary for vertical immersion of a blade under load in a rock mass layer was studied.

The work aimed to determine the impact energy necessary for immersing the blade in the rock mass at a given depth $\mathrm{h}$ depending on the particle size and moisture content of the raw material.

\section{Methodology}

To achieve this goal, experiments were conducted and on their basis mathematical models were developed for determining the impact energy depending on the influencing factors.

For the experiments, a stand was made, consisting of a box with a rock mass above which there is a movable frame with clamps for the blade with a load fixed on it. Using the frame, a different height of the fall of the blade was set. A strain gauge was placed under the bottom of the box to measure the force and time of the impact.

When conducting experiments under the influence of gravity, the blade with the load hits the rock mass. Depending on the thickness of the layer $h$, particle size $d$ and humidity $W$, the experimenter selects a drop height $H$ (directly proportional to the impact energy $E$ ) 
that allows the blade to penetrate completely through the entire rock mass layer. This height of incidence $N$ is fixed.

Knowing the mass of the blade with the load and the height of the fall $H$, the impact energy is defined as $E=m g \cdot H$, where $g=9.8 \mathrm{~kg} \cdot \mathrm{m} / \mathrm{s}^{2}$. In the experiments, the mass of the blade with the load was $m=0.945 \mathrm{~kg}$, that is, $E$ and $H$ are connected as: $E=9.261 \cdot N$, where $E$ is in joules, $N$ is in meters.

The research material was crushed granite. In the experiments, the layer thickness varied: $20 ; 30$ and $40 \mathrm{~mm}$. Four classes of particle size in the layer were studied: $(-2.5+1)$ $\mathrm{mm}$, an average of $1.75 \mathrm{~mm} ;(-5+2.5) \mathrm{mm}$, an average of $3.75 \mathrm{~mm} ;(-7+5) \mathrm{mm}$, an average of $6 \mathrm{~mm}$ and $(-10+7) \mathrm{mm}$, an average of $8.5 \mathrm{~mm}$. Next, the indicated average value for each class is used.

The moisture content of the material changed from the initial, which was conditionally considered zero and then designated as $W=0$, to the values of $W=3 ; 6$ and $9 \%$. For the initial humidity, relative to which the measurements were carried out, $W$ was taken as raw material upon exposure for 14 days in laboratory conditions at room temperature.

A base of experimental measurements of impact energy $E$ was obtained, in which one of the parameters $h, d$, or $W$ was varied, the other two were fixed. The database was processed by methods of correlation and regression analysis using free distributed programs Microsoft Excel and SPSS Statistics [5]. The accuracy of the approximation was estimated by the squared correlation coefficient $R^{2}$.

\section{Results and discussion}

Figure 2 shows the dependences of the impact energy of the blade on the particle size and the thickness of the granite layer for the initial moisture content $W=0$. Figure 2 shows that at $W=0$, the larger the particles and the thicker the layer, the higher should be the impact energy. For dependencies Figure 2 constructed correlation equations.

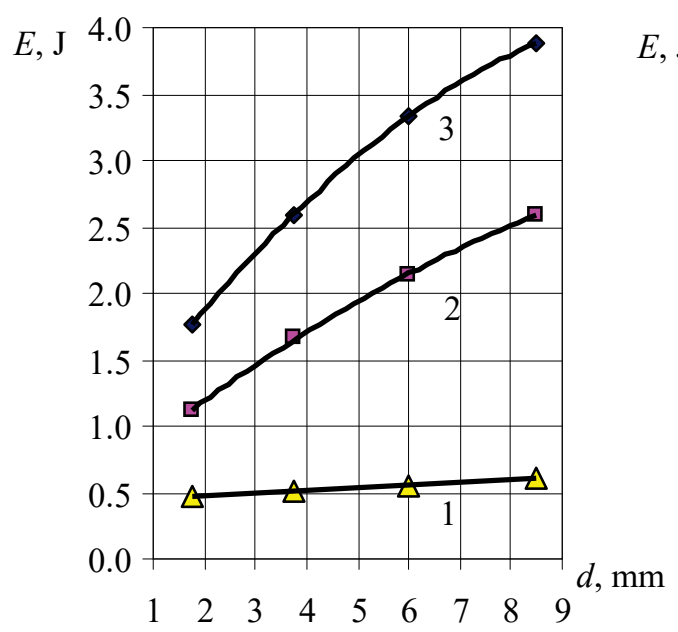

$1-h=20 ; 2-h=30 ; 3-h=40(\mathrm{~mm})$

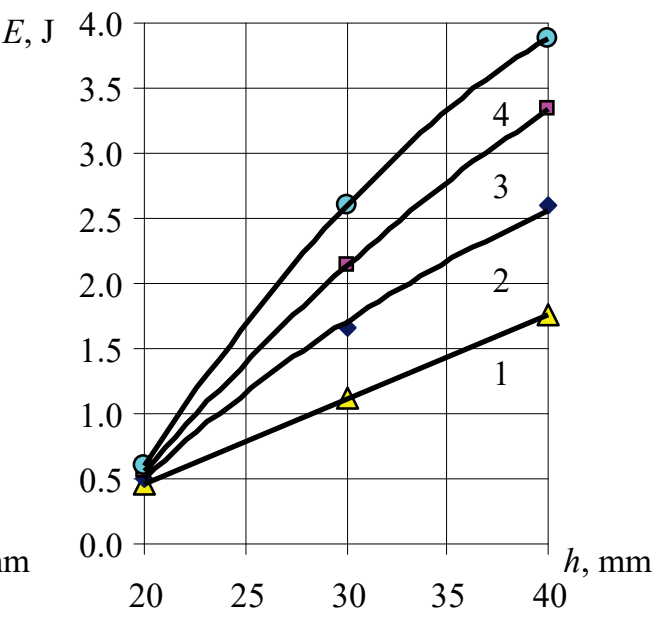

$1-d=1.75 ; 2-d=3.75 ; 3-d=6$; 4- $d=8.5(\mathrm{~mm})$ $\mathrm{b}$

a

Fig. 2. The dependence of the impact energy of the blade on the particle size (a) and layer thickness (b) for the initial moisture content of the raw material. 
For dependencies Figure 2 (a), when $W=0$, received:

$$
\begin{aligned}
& E=0.02 \cdot d^{2}+0.54 \cdot d+0.88, R^{2}=0.99, h=40 \mathrm{~mm}, \\
& E=0.01 \cdot d^{2}+0.32 \cdot d+0.59, R^{2}=0.99, h=30 \mathrm{~mm}, \\
& E=0.02 \cdot d+0.43, \quad R^{2}=0.99, h=20 \mathrm{~mm} .
\end{aligned}
$$

For dependencies Figure 2 (b), when $W=0$, approximating equations are obtained:

$$
\begin{aligned}
& E=0.003 \cdot h^{2}+0.373 \cdot h-5.464, R^{2}=0.99, d=8.5 \mathrm{~mm}, \\
& E=0.0019 \cdot h^{2}+0.253 \cdot h-3.704, R^{2}=0.99, d=6 \mathrm{~mm}, \\
& E=0.0012 \cdot h^{2}+0.174 \cdot h-2.5, \quad R^{2}=0.99, d=3.75 \mathrm{~mm}, \\
& E=0.0648 \cdot h-0.833, \quad R^{2}=0.99, d=1.75 \mathrm{~mm} .
\end{aligned}
$$

A comparison of the coefficients of the correlation equations shows that the magnitude of the coefficients increases with increasing layer thickness - formula (1) and particle size formula (2), that is, the impact energy increases.

Note that with decreasing particle size and layer thickness, the dependences of Figure 2 are flat out. Equations (1), (2) show that for small size $d=1.75 \mathrm{~mm}$ and small layer thickness $h=20 \mathrm{~mm}$, the dependences are approximated by a linear function rather than a quadratic one. Whereas for larger particles and a thicker layer, the dependence of the impact energy on $d$ and $h$ is nonlinear. It is described with high accuracy $\left(R^{2} \sim 1\right)$ by a polynomial of the second degree.

Such nonlinearity was taken into account when constructing regression models of the dependence of $E$ on all influencing factors. For this, was used the free-distributed statistical data processing program SPSS Statistics [5].

At the first stage, the influence of variable factors on $E$ at the initial moisture content of the raw material $W=0$ was studied. First, an approximate linear regression equation without a free term was obtained, which has the form $E=f(d, h)$ :

$$
E=0.069 \cdot d+0.052 \cdot h, R^{2}=0.886, W=0 .
$$

Equation (3) allows us to estimate the degree of influence of each factor, despite the insufficiently high correlation for $R^{2}$. So, judging by the magnitude of the coefficients of equation (3), the effect of $d$ on energy is slightly higher than $h$. For the initial humidity content $W=0$ of crushed granite, the influence of particle size and layer thickness on the energy of penetration into the layer is correlated in percent as $d: h=57: 43$.

Further, for $W=0$, a generalized linear regression equation with higher approximation accuracy is obtained. It has the form $E=f\left(C, d, h, d^{2}, d \cdot h, h^{2}\right)$, where $C=$ const is a free term.

$$
\begin{gathered}
E=-2.054-0.147 \cdot d+0.142 \cdot h-0.011 \cdot d^{2}+0.015 \cdot d \cdot h-0.002 \cdot h^{2}, \\
R^{2}=0.996, W=0 .
\end{gathered}
$$

At the second stage, the influence of humidity on the impact energy was investigated. Figure 3 shows the dependence of $E$ on moisture for two classes of fineness - the largest $8.5 \mathrm{~mm}$ (Figure 3.a) and the smallest $1.75 \mathrm{~mm}$ (Figure 3.b). 


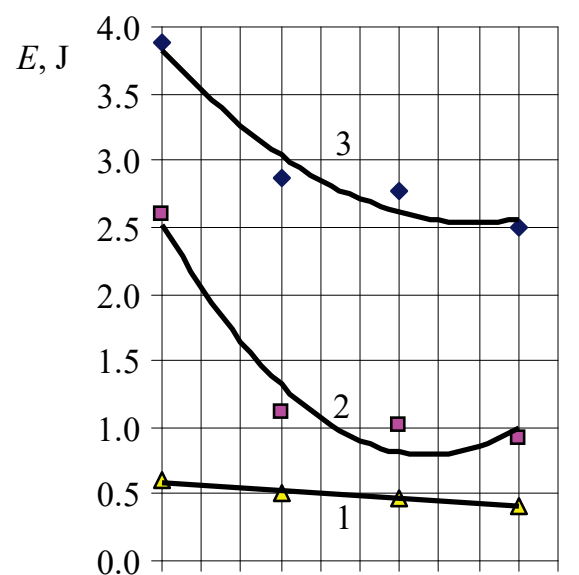

$\begin{array}{lllllllllllll}0 & 1 & 2 & 3 & 4 & 5 & 6 & 7 & 8 & 9 & 10 & \mathrm{~W}, \%\end{array}$

$$
d=8.5 \mathrm{~mm}
$$

$1-h=20 ; 2-h=30 ; 3-h=40(\mathrm{~mm})$

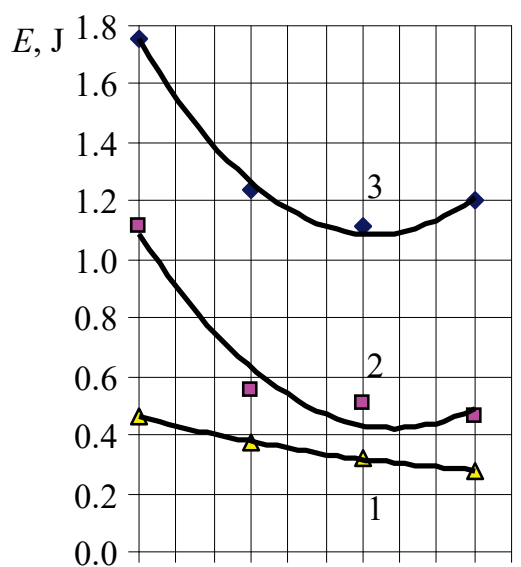

$\begin{array}{llllllllllll}0 & 1 & 2 & 3 & 4 & 5 & 6 & 7 & 8 & 9 & 10 & \mathrm{~W}, \%\end{array}$

$$
d=1.75 \mathrm{~mm} \text {. }
$$

$1-h=20 ; 2-h=30 ; 3-h=40(\mathrm{~mm})$

b

Fig. 3. Dependence of impact energy on humidity at different layer thicknesses with particle size.

A comparison of Figures 3.a and 3.b shows that for the same layer thickness, for any humidity, large particles require a higher impact energy than for small ones. The decreasing nature of the $E(W)$ dependences in Figure 3 shows that an increase in humidity facilitates the penetration of the blade - the process requires less energy. This is due to a decrease in external friction with increasing humidity.

The correlation equations of dependencies in Figure 3 have the form:

- for $d=8.5 \mathrm{~mm}$ :

$$
\begin{gathered}
E=0.021 \cdot W^{2}-0.327 \cdot W+3.834, R^{2}=0.94, h=40 \mathrm{~mm}, \\
E=0.039 \cdot W^{2}-0.517 \cdot W+2.524, R^{2}=0.95, h=30 \mathrm{~mm}, \\
E=-0.002 \cdot W+0.588, \quad R^{2}=0.966, h=20 \mathrm{~mm},
\end{gathered}
$$

-for $d=1.75 \mathrm{~mm}$ :

$$
\begin{aligned}
& E=0.023 \cdot W^{2}-0.261 \cdot W+1.718, R^{2}=0.90, h=40 \mathrm{~mm}, \\
& E=0.014 \cdot W^{2}-0.194 \cdot W+1.086, R^{2}=0.95, \quad h=30 \mathrm{~mm}, \\
& E=-0.002 \cdot W+0.449, \quad R^{2}=0.966, \quad h=20 \mathrm{~mm} .
\end{aligned}
$$

A feature of equations (5), (6) is that for a small layer thickness $h=20 \mathrm{~mm}$ with sufficient accuracy, $R^{2}=0.966$, a linear approximation can be used for both small classes of $1.75 \mathrm{~mm}$ and large classes of $8.5 \mathrm{~mm}$. From equations (2) it can be seen that the function $E(h)$ for small classes of $1.75 \mathrm{~mm}$ is also linear. Given this, for small classes we obtain a simple generalized regression equation for the dependence of $E$ on two variables $E=f(h, W)$. At the same time, we assume that the free term (constant) is equal to zero. The analysis showed that, compared with the equation with a nonzero constant, this increases 
the accuracy of the approximation in the estimate with respect to $R^{2}$, and also makes it possible to more clearly compare the coefficients.

For the smallest classes of fineness $d=(-2.5+1) \mathrm{mm}$, on average $1.75 \mathrm{~mm}$, the dependence is obtained:

$$
E=0.035 \cdot h-0.056 \cdot W, \quad R^{2}=0.944
$$

From equation $(7)$ it follows that for classes $(-2.5+1) \mathrm{mm}$, the influence of humidity on the impact energy exceeds the influence of the layer thickness. In percent, they are correlated as $61.5: 38.5$. In this case, an increase in the layer thickness requires an increase in impact energy, and an increase in humidity leads to its decrease.

To take into account the influence of all three variable factors on the impact energy, an approximate generalized regression equation was first obtained in the form $E=f(C, d, h, W)$. Taking $C=0$, we get:

$$
E=0.078 \cdot d+0.049 \cdot h-0.123 \cdot W, \quad R^{2}=0.868 .
$$

Equation (8) is considered approximate due to the insufficiently high value of $R^{2}$, but it allows us to estimate the influence of each of the factors. So, the sign with the coefficients shows that an increase in the fineness and thickness of the layer leads to an increase in energy, and an increase in humidity decreases it.

Judging by the magnitude of the coefficients, the greatest influence on the impact energy is exerted by the moisture of the material, and the higher the humidity, the less energy is needed. In second place - particle size, in third - layer thickness, the larger these parameters, the greater the energy needed to immerse the blade in a layer of crushed mass. According to the degree of influence, the factors are correlated in percent as $d: h$ : $W=31.2: 19.6: 49.2$.

The equation has the form $E=f\left(C, d, h, W, d^{2}, d \cdot h, d \cdot W, h^{2}, h \cdot W, W^{2}\right)$, where the free term $C$ is taken equal to zero to achieve a higher value of $R^{2}$ ( 0.985 vs 0.96 with a constant). Received:

$$
\begin{gathered}
E=-0.0135 \cdot W-0.0092 \cdot d-0.022 \cdot h+0.0161 \cdot W^{2}-0.0098 \cdot W \cdot d- \\
-0.0053 \cdot W \cdot h-0.016 \cdot d^{2}+0.0116 \cdot d \cdot h+0.0013 \cdot h^{2}, \quad R^{2}=0.985
\end{gathered}
$$

The obtained correlation and regression equations make it possible to determine the impact energy of the blade of the roller vibratory classifier depending on the particle size, humidity and rock mass thickness, which is necessary to improve the existing design and develop new device models.

\section{Conclusions}

At the initial humidity content of crushed granite (conventionally $W=0$ ), it is established that the impact energy required for the penetration of the blade into the rock mass layer is influenced by the size of the raw material slightly higher than the influence of the layer thickness, their percentage is $d: h=57: 43$. It was found that an increase in humidity leads to a decrease in impact energy, which is a consequence of a decrease in the influence of external friction forces, while an increase in particle size and layer thickness requires an increase in energy.

For small classes of $(-2.5+1) \mathrm{mm}$, a linear approximation of the dependence of the impact energy on the change in humidity and layer thickness was obtained. It is established 
that for the indicated small classes the influence of humidity on energy prevails and in a percentage ratio is $W: h=61.5: 38.5$.

It is determined that the percentage of all three variable factors on the impact energy is $W: d: h=49.2: 31.2$ : 19.6 . It can be used to introduce correction coefficients when calculating the energy characteristics of a blade-type roller vibratory classifier taking into account moisture, particle size and rock mass thickness.

For practical application in the design of blade-type roller vibratory classifiers, the following regression models of impact energy changes from influencing factors are recommended: model (3) for initial humidity, model (7) for small classes $(-2.5+1) \mathrm{mm}$ and (9) generalized model when changing parameters in the range $W=0-9 \%, d=1-10 \mathrm{~mm}$, $h=20-40 \mathrm{~mm}$. The obtained models have a high accuracy of the approximation and are recommended for use in the design and determination of the parameters of blade-type roller vibratory classifiers of different sizes.

\section{References}

1. Nadutyi, V.P, Egurnov, A.I., Yagnyukova, I.V. (2013). Modernization of the vibrational roll classifier based on the use of vibration-shock mode, Visnyk NTU "KhPI", 57 (1030), 89-96

2. Nadutyi, V.P., Iagniukov, V.F., Iagniukova, I.V. (2015). Sposob klassifikatsii trudnoobogatimogo mineralnogo syria [Method of classification of hard-to-sieve mineral materials] Patent No 108453, Ukraine

3. Nadutyi, V.P, Jamil Haddad, Sukhariev, V.V. (2018), Investigation of the process of destruction of a layer of mined rock by the toothed disc of the roller classifier. Proceedings of the XVII International Science-Techn. Conf. "Vibration in Engineering and Technology", Nat. University "Lviv Polytechnic", Lviv

4. Nadutyi, V.P, Yagnyukov, V.F., Yagnyukova, I.V. (2017). Productivity of roll vibroimpact classifier depending on design and operating parameters based on experimental studies. Proceedings of the Ukraine Science-Techn. Conf., National Metallurgy Academy, Inst. of Black Metallurgy, 46-48

5. Buyul, A., Tsefel, P. (2005). SPSS: the art of information processing. Analysis of statistical data and restoration of hidden patterns. St. Petersburg, Russia, 608 